\title{
Advanced matrix laser lithography for fabrication of photonic micro-structures
}

\author{
Marek Škereň \\ marek.skeren@fjfi.cvut.cz \\ Jakub Svoboda \\ Pavel Fiala
}

\author{
Faculty of Nuclear Sciences and Physical Engineering, Czech Technical University in Prague, Břehová \\ 7, 11519 Prague 1, Czech Republic \\ Faculty of Nuclear Sciences and Physical Engineering, Czech Technical University in Prague, Břehová \\ 7, 11519 Prague 1, Czech Republic \\ Faculty of Nuclear Sciences and Physical Engineering, Czech Technical University in Prague, Břehová \\ 7, 11519 Prague 1, Czech Republic
}

Direct laser beam writing techniques represent an interesting alternative to focused electron and ion beam writing approaches for the fabrication of photonic micro-structures. Although the resolution of laser beam writers is strongly limited by the Rayleigh diffraction limit, it is not always necessary to create features significantly smaller than the recording wavelength. In such cases, a laser exposure can be used, with all its advantages. In this paper a direct laser writing technique is presented one; which significantly improves the performance of commonly used direct laser writers by introducing an exposure of a large area within a single step. The elementary exposed area can contain a general micro-structure without any special requirements. In contrast to writing techniques based on a single spot focus, the writing beam can have the shape of a general aperiodic structure and exposes an area of about $10^{-2}$ square millimetre. This complicated exposure field is created using a demagnified projection of the exposed structure from a two-dimensional computer-driven spatial light modulator. The main advantages of the method compares to the single point exposure are a significantly improved exposure speed, a perfect alignment of features within a single exposed area, and the possibility of tuning the exposing field very precisely using continuous modulation of the signal on the spatial light modulator. The in-house-developed device is presented together with several realized samples. [DOI: http://dx.doi.org/10.2971/jeos.2012.12043]

Keywords: Diffractive optics, laser lithography, direct laser writing, photo-resist material

\section{INTRODUCTION}

Today, the photonic structures of various types play an important role in many fields of optics. These elements offer several interesting properties, which can be utilized in particular applications such as optical sensors, optical communications, optical spectroscopy, biology, and others. Recent applications often require complicated micro-structures with an elementary feature size comparable to a wavelength of light, which have a general three dimensional geometry. Thus there is a need for experimental techniques capable of preparing such elements with reasonable speed and costs. The crucial parameters of the structures realized are spatial resolution and the total area (or volume). The leading-edge lithographic devices have been primarily developed for the semiconductor industry [1]-[3]. However, these techniques also play an important role in other areas such as medical devices [4]-[6], diffractive structures [7], photonic crystals [8]-[10], etc [11]. The most common techniques for creating diffractive and photonic structures are electron beam lithography [12], ion beam lithography [13] or optical lithography [14]. Electron and ion beam lithography can achieve resolution down to the nanometric scale (feature size $<5 \mathrm{~nm}$ ), which beats most of the other current techniques. However, application of these techniques is often complicated (because of high initial price and high operation costs, limited size of samples, etc.). Unless a modern variable shaped e-beam system is used [15], exposure speeds are very low. From a point of view of photonic structures, an even more important issue is the limited applicability of these techniques for three dimensional structures (blazed gratings, 3D photonic crystals, etc).

Large amounts of micro/nano structures are created using direct writing optical lithography approaches [16]-[18]. These methods can often achieve a considerably higher exposure speed and they can also be more versatile. The resolution limit of the laser lithography is derived from the Rayleigh diffraction limit. Therefore, the higher resolution is often acquired using a laser source with a shorter wavelength (the deep UV lithography, etc.) [19]. Exposure of larger areas is well mastered in dot-matrix and general matrix laser lithographic techniques [20,21], which have been recently used in the field of synthetic image holograms for applications in optical document security. Modification of these approaches for the fabrication of photonic micro/nano structures can lead to techniques capable of realizing the desired features rapidly and cost-effectively over larger areas. Some of these recent approaches use multiple foci from a microlense array to speed up the single-focus process [22], while others have shown the possibility of creating multiple foci using a synthetic hologram displayed on a spatial light modulator (SLM) [23, 24]. The SLM can be also used for imaging the structure directly 
onto recording material [25]-[27]. Another approach for creating the micro/nano structures in larger areas (or volumes) is the use of multiple broad beam interference lithography $[28,29]$.

There are also a few commercially available devices. Besides the initial price, the main disadvantage of the commercial systems is their very limited adaptability to the needs of particular experiments when they are not used directly for their primary intended application. Therefore, the main focus of this work is on an in-house built device, which can be easily adapted to the current needs of the research group. There are several interesting devices available, dedicated to particular applications. The laser direct imaging systems by $\mathrm{Mi}$ cronic [25] offer high throughput manufacturing of microstructures for the electronics industry (the feature size is below 10 micrometres). Heidelberg Instruments Mikrotechnik offers mask-less laser lithography systems for micro and nano technology, micro optics, etc. (the feature size is down to $600 \mathrm{~nm}$ ). The set-up closest to the device presented is used in the Kinemax ${ }^{T M}$ from Polish Holographic Systems [30] (but this device is intended rather for the area of optical document security and does not comply with the specific demands for photonic micro/nano structures).

In this paper a matrix laser writing technique which significantly improves the writing speed and also the robustness of a positioning system is presented and one which enables us to expose fine micro-structures over large areas at high speed and at a moderate cost. Instead of a single spot, a larger area is exposed within a single exposure (typically tens of thousands of square micrometres). The experimental setup is discussed in detail. In comparison with current devices, the stage positioning system and the optical focusing system have been improved together with the driving software. Several samples are also presented, which were prepared using the developed device.

\section{MATRIX LASER LITHOGRAPHY}

There are three important parameters which influence the applicability of the direct writing laser technique for very fine micro-structures. The first one is the size of the focal spot which limits the dimensions of the smallest features that can be created within a single shot. The principal limitation of the focal spot size follows from the Rayleigh diffraction limit and is given by the wavelength of the light source and the numerical aperture of the optical system. The second important parameter, which limits the achievable resolution, is the positioning accuracy of subsequent exposures. In contrast to direct writing with focused electron or ion beams, where the writing beam can be positioned using electrostatic or electromagnetic deflectors, laser writers usually move the sample stage instead of the beam itself. The current stage positioning systems based on piezoelectric actuators can easily reach nanometer resolution. The last important parameter of the laser writing system is its exposure speed. Because of an extremely small spot size and high requirements concerning precise positioning, the exposure of a reasonable area of several square millimetres can take a long time.

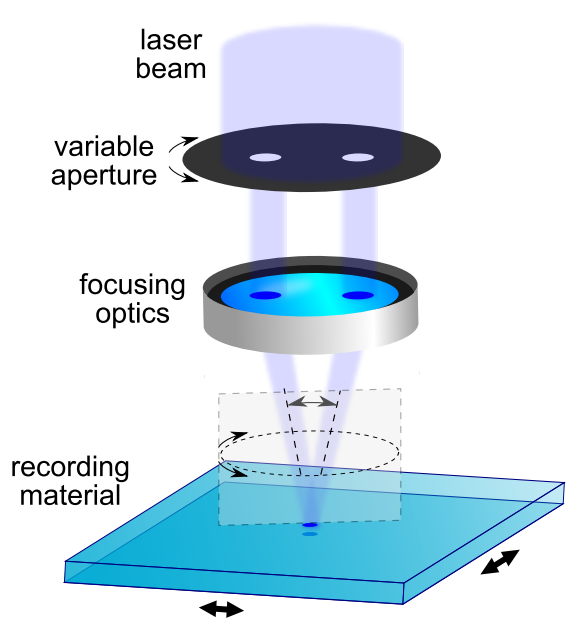

FIG. 1 The basic setup of a simple dot-matrix writer with two writing beams. A single micro-grating is exposed within one step. The parameters of the recorded micro-grating can be changed by rotating the plane of incidence of the beams and by changing their respective angle. In this example this is accomplished by a double aperture in a rotating screen with a variable distance of apertures.

In the field of synthetic image holography so-called dotmatrix devices are commonly used to create a system of regular micro-gratings. If the elementary exposed area contains only a regular grating, a natural process of interference of two focused laser beams can be used. In Figure 1 the setup of such a dot-matrix device can be seen. The profile of the grating is predetermined by the two beam interference process and thus it is harmonic (however, when a non-linear recording material is used, the recorded structure can also have a different profile). The periodicity and orientation of the grating modulation can be changed by setting the respective angle of the recording beams and by changing the orientation of their plane of incidence. This can be done for example by using a mechanically variable double aperture, as is indicated in Figure 1. The main advantages of this approach are the rapid increase of the writing speed and the low sensitivity of the exposure field to various disturbances. The total area, which is exposed within a single matrix exposure can be defined according to the particular application. Because the exposed micro-structure is strictly regular, the recorded elements have a strongly limited variability of spatial features. Moreover, it is usually impossible to precisely align subsequent exposures on a sub-wavelength scale as the phase of the interference field is not perfectly controlled.

The interference technique can also be used for writing aperiodic micro-structures if more than two beams are used. When an arbitrary number of interfering beams is assumed, the recorded structure can be general. More precisely, the decomposition of the exposure field to a system of plane waves can be done by the Fourier transform of the desired shape of the structure. However, it can be technically complicated to set up such a system of writing beams. When the advanced matrix approach is used, the recording beams are created using diffraction from a spatial light modulator. In fact, the whole process can be interpreted as a projection of the recorded element from a micro-display to a recording material accompanied with a strong de-magnification. The basic idea of such a projection is depicted in Figure 2. 


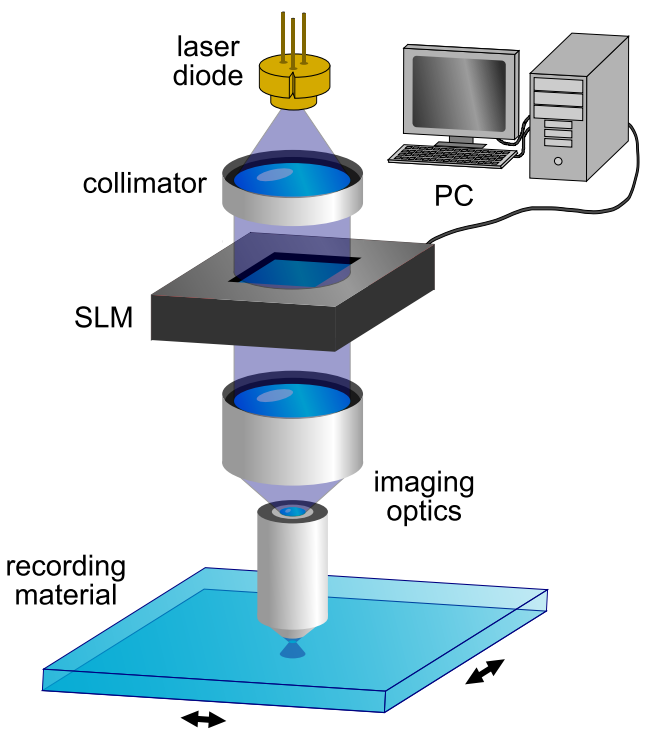

FIC. 2 The basic setup of the advanced matrix laser writing system. The recorded structure is projected from a computer driven spatial light modulator using a demagnifying optical system directly onto the surface of a recording material.

A spatial light modulator is illuminated with a collimated laser beam. An optical system is used to de-magnify the modulator to the desired size and to image the recorded microstructure onto a recording material. The recorded structure can be general, both periodic and aperiodic shapes can be displayed. For applications in synthetic image holography a hybrid structure can be used consisting of a system of different micro-gratings projected side-by-side from the modulator to the recording material.

The optical resolution of the writing system is limited by several parameters. Again, the Rayleigh diffraction limit restricts the imaging process. However, the recorded micro-structure is also influenced by the elementary pixel size of the spatial light modulator and by the magnification of the imaging system. The final dimensions of a single projected addressable pixel from the modulator can be well beyond the diffraction limit (if the pixel size on the modulator is sufficiently small and the de-magnification of the optical system is high). Although a single pixel cannot actually be imaged, it can still be meaningful to use such a configuration, as the projected pixel size also simultaneously determines the positioning precision of the elementary features over the recorded element's area. This property can be used to some advantage for exposing grayscale images to achieve continuous 3D reliefs.

The application of the two-dimensional computer-driven spatial light modulator brings several important improvements. The recording speed is further increased compared to the two-beam dot-matrix systems, because more than one micrograting can be exposed within a single exposure (typically, when recording the synthetic image holograms, several hundreds micro-gratings are exposed in a single shot). For more general micro-structures, the ability of the technique to perfectly position the details within a single exposure area is much more important. As the object on the modulator is mechanically stable, relative positioning within the exposure field is very precise (the only important distortion can be caused by imperfections of the optical imaging system). It can also be useful to expose the same area more than once with different patterns. When elements with fine details are exposed, all subsequent exposures must be perfectly aligned. This can be easily done with the setup presented.

\section{EXPERIMENTAL SETUP}

The matrix writing device, which is based on the principles presented, has been built at the department. Because the main purpose of the system is the preparation of various microstructures with a high flexibility of shape and size, the main focus was on the parameters influencing the precision of the system, particularly the optical setup, the positioning system, and focusing system.

\subsection{OPTICAL SETUP}

The optical setup of the device is displayed in Figure 3. As a light source, the laser diode Nichia NDV4313 was used, operating on the wavelength $405 \mathrm{~nm}$ with a typical optical output power $120 \mathrm{~mW}$. The operating temperature of the diode is stabilized with the Thorlabs TCLDM9 cooled diode mount. The diode is powered with the Thorlabs ITC110 controller, which also provides the power feedback and can actively stabilize the output power of the laser. The light from the laser is collimated and it illuminates the spatial light modulator. The Holoeye LC-R 1080 spatial light modulator was used for projecting the input data. The modulator is a reflective liquid crystal on silicon (LCoS) based device with a high resolution (1920 × 1080 pixels) and a high contrast operating in an amplitude modulation regime. The elementary pixel size is $8.1 \mu \mathrm{m}$ and the fill factor of the display is $90 \%$. The device operates with a refresh rate $60 \mathrm{~Hz}$ and is addressed through a digital visual interface (DVI). Because of the operation in a reflective regime, there is a polarizing beam splitter cube in front of the modulator, which separates the incident and the diffracted beams. Finally, the image from the modulator is demagnified in an optical system which consists of two objectives. The first objective is the photographic lens Carl Zeiss Sonnar with a focal length of $300 \mathrm{~mm}$ and a maximum aperture of 4 . The second objective is the microscope objective Mitutoyo M Plan APO HR 50× with the numerical aperture 0.75 and the working distance $5.2 \mathrm{~mm}$. In the setup from Figure 3 the total de-magnification of the system is given by the ratio of the focal lengths of both objectives $(75 \times)$. The Rayleigh limit for the microscope objective and the used wavelength is $\sim 300 \mathrm{~nm}$, the theoretical projected elementary pixel size at the recording material's surface is $\sim 100 \mathrm{~nm}$.

In Figure 3 there is also a spatial filter placed at the front focal plane of the microscope objective, which can further modify the exposure field. In a simple case a circular high-pass filter can be used, which blocks the low spatial frequencies of the field and transmits only higher frequencies. When a system of regular micro-gratings is recorded, such a filtration can rapidly improve the robustness of the system and decrease the sensitivity to proper focusing. Moreover, this operation automatically doubles the grating frequency, which helps to write 


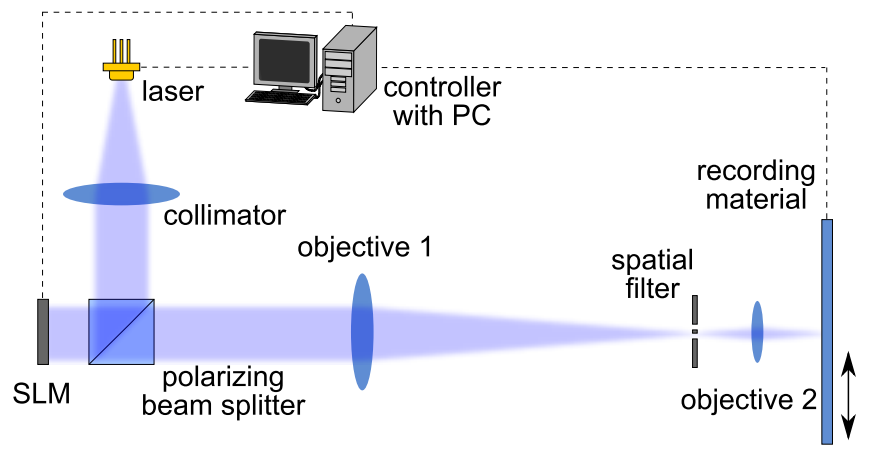

FIC. 3 Basic optical setup of the matrix writing device. A collimated, linearly polarized laser beam illuminates the image on the modulator which is further de-magnified using a system of objectives. In the chosen geometry, the overall de-magnification of the system is given by the ratio of focal lengths of objectives 1 and 2 .

grating lines with higher densities. Unfortunately, the spatial filtering can also blur sharp edges in projected images, especially for aperiodic patterns.

The filtering, when used, can also help to improve the quality of the exposing wavefront, as the noise is filtered out together with unwanted orders. However, for aperiodic structures, the spatial filter must be removed and it could be necessary to apply spatial filtering of the beam directly in front of the laser diode. Both setups were successfully tested. The image quality is also influenced by coherent noise (speckle), which can negatively affect the surface roughness of the exposed element. For some applications, this can be avoided by decreasing the coherence of the source (either by using a different light source or by adding some kind of diffusor).

\subsection{STAGE POSITIONING SYSTEMS}

The positioning precision of the matrix writers within a single exposure is also very high for relatively simple systems. However, when a large area element is recorded, many exposures must be performed with a precise stage movement between subsequent exposures. Because of the positioning precision $\sim 100 \mathrm{~nm}$ within a single exposure area, two M-511.HD Ultra High Resolution stages from Physik Instrumente were used to reach a comparable value, likewise for the mechanical stitching. The stages are based on a combination of an electric servomotor with a piezoelectric actuator. The incremental step of the piezoelectric actuator is $4 \mathrm{~nm}$. The linear encoder with the resolution $2 \mathrm{~nm}$ gives an accuracy of below $50 \mathrm{~nm}$ with a repeatability of $10 \mathrm{~nm}$. These parameters can be used within a travel range of $100 \mathrm{~mm}$ with a maximum velocity of $125 \mathrm{~mm} / \mathrm{s}$. Two stages with these specifications were crossed to form a two dimensional xy positioning system with a total covered area of $100 \mathrm{~cm}^{2}$.

The positioning system was tested in an experiment which is described in Figure 4. A grating with periodicity $600 \mathrm{~nm}$ was projected onto the photo-resist surface and exposed. Then the stages moved along a rectangle with the dimensions $20 \times 20 \mathrm{~mm}$, returned back to the original position and finally moved to the position of an adjacent exposure. In Figure 4, there is an AFM scan of a border area between the two grat-

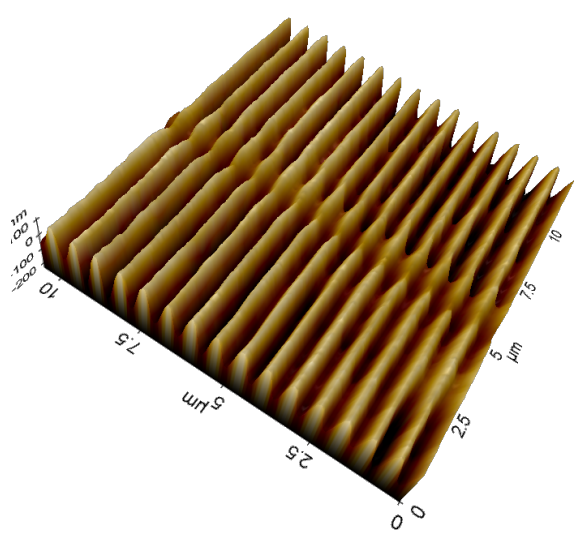

FIG. 4 Demonstration of the stitching accuracy of the positioning system. An AFM scan of two adjacent gratings with the period $600 \mathrm{~nm}$. The second grating was exposed after long range travel of both stages from the position of the first grating and back. The stitching error is small compared to the periodicity of the grating and also much smaller than the image positioning accuracy given by the projected resolution of the modulator

ings. Although the border is clearly visible, the synchronization of the gratings is almost perfect and definitely better than the projected pixel size $100 \mathrm{~nm}$.

The precise stitching of the neighbouring exposures does not necessarily imply absolute precision over the whole travel range of the stages. So far absolute precision has not been evaluated (the stages were only tested separately in an experiment with an external interferometer for linearity of movement). For tested applications of the device it was not crucial to achieve such precise stitching over the whole area of the optical element. However, absolute precision can be important for the wavefront quality of large area holographic elements and other structures. For the quality of the written elements, it is also necessary to adjust the mutual perpendicularity of the stage axes and the perpendicularity of the SLM image to these axes. This was ensured by an adjusting system, where the angle between the stages was set using a micrometric screw and a lever. The SLM was mounted in a commercial adjustable mount. A series of testing exposures were performed and evaluated using the AFM and the position of stages was iteratively tuned.

\subsection{DYNAMIC FOCUSING SYSTEM}

The theoretical limits for the elementary feature size were derived under the assumption of perfect focusing of the imaging system. However, the proper focusing is a tricky task, especially when large area structures are exposed. Two focusing systems were proposed and tested in the device developed, one passive with a back reflection of the projected image and one active with a dynamic evaluation of the focus state and a correction for distortions during the exposure.

The passive focusing system was successfully tested with a transmissive spatial light modulator (Holoeye LC2002). It is described in Figure 5. A test pattern is displayed onto the modulator and projected onto the plane of the recording material in laser light. Then it is reflected (either by the photo-resist surface itself or by a mirror placed at the recording plane). 


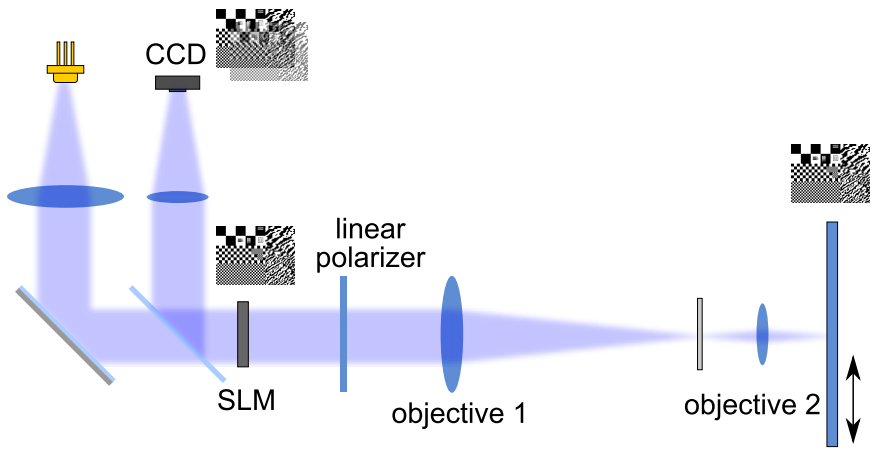

FIG. 5 Main principle of the passive focusing system. An image from the modulator is reflected from the recording material and is observed again on the modulator's surface using a CCD camera. The overlap of the original image and its reflected copy is evaluated. The direct evaluation of the image reveals most distortions of the imaging system.

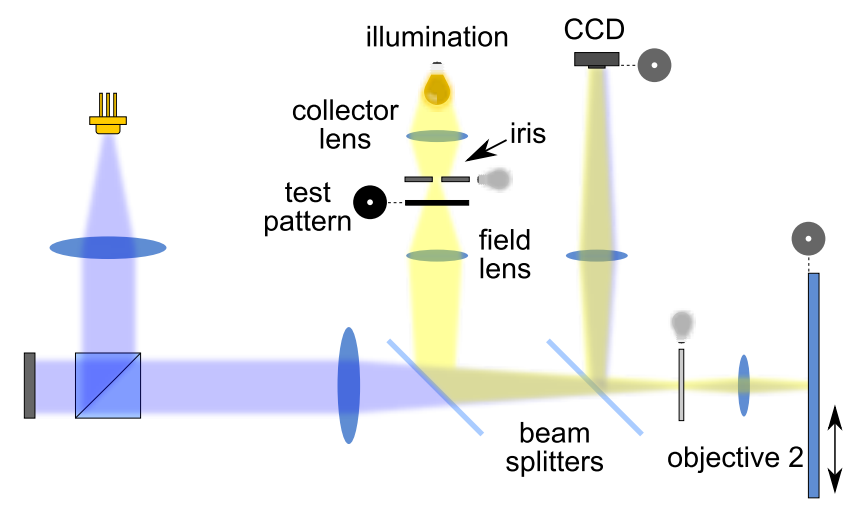

FIG. 6 The dynamic focusing is based on an auxiliary projection of a test pattern from an illumination system to the surface of a recording material. The test pattern (for example a pinhole aperture) is projected onto the recording plane and the projection is observed by a CCD camera. The main projection system is first focused together with the focusing pattern and the CCD camera onto the recording plane. During the exposure, the image of the pinhole is used as a reference for the focusing system and any distortion in the projection is compensated by moving the objective 2 .

The image is propagated back to the spatial light modulator and is evaluated with a CCD camera. If the system is properly focused, the reflected image perfectly overlaps the image on the modulator otherwise the distortion can be corrected by moving the microscope objective. However, there are several important drawbacks. The method seems to be difficult to use with reflective modulators because of the complicated observation of the reflected image due to the presence of the polarizing beam-splitter cube. Moreover, the reflected image is usually very weak compared to the image on the modulator. Finally, also, when focusing is possible directly on the recording material, distortions can occur during the exposure because of improper flatness of a substrate. This effect is more significant, when larger areas are exposed. If the focusing state is not controlled dynamically during the exposure, other effects can also bring the system out of focus (for example there is a strong temperature dependency of the focus position, etc.)

Because of the issues mentioned, the dynamic focusing system was introduced. The basic setup of the system is displayed in Figure 6. The system uses an independent light source for the projection of a test pattern, which can be observed continu-

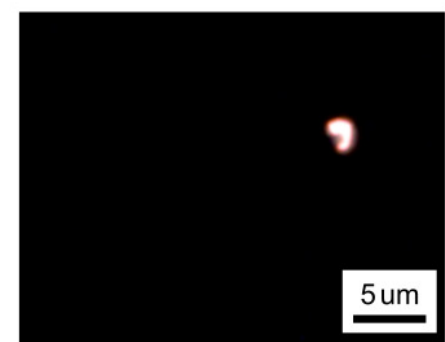

(a)

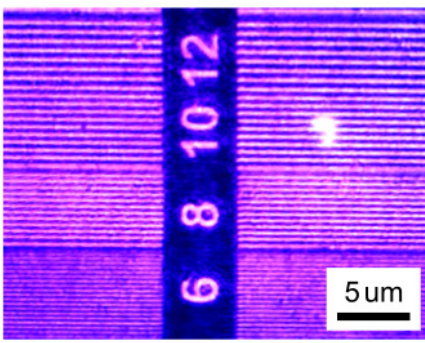

(c)

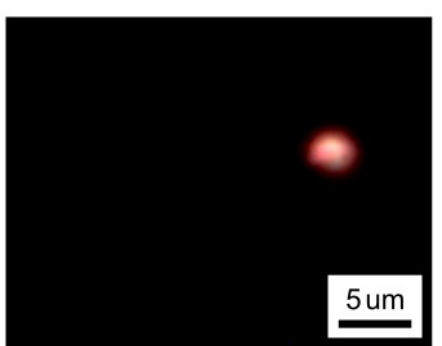

(b)

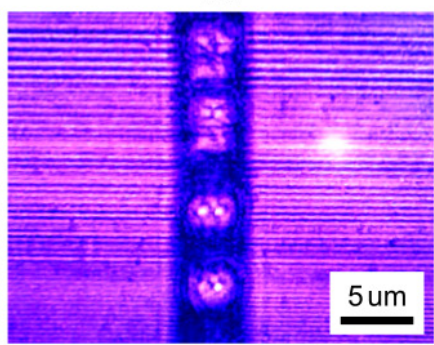

(d)
FIG. 7 Examples of images from the focusing system observed by the CCD camera. Images (a) and (c) correspond to the focused system, images (b) and (d) correspond to the system slightly out of focus. Images (a) and (b) display the projected test pattern (aperture), images (c) and (d) show the corresponding exposure fields projected from the modulator.

ously during the exposure. A white light source was used in combination with an edgepass filter to avoid exposure of the recording material with an auxiliary beam.

The test pattern itself is placed in the Kohler illumination setup directly at the front focal plane of a field lens (see Figure 6). The image of this pattern is projected onto the plane of the recording material and is observed with a CCD camera focused on the same plane. If there is any distortion of the focus caused by the improper flatness of the substrate or by other disturbances, the image of the test pattern is blurred. The microscope objective is attached to a piezo-electric actuator, which is connected to a feedback loop together with a CCD camera and driving software. When such a distortion occurs, the feedback loop moves the objective and compensates for this distortion. The feedback is based on evaluation of the image of the test pattern. When a proper test pattern is chosen, the peak intensity can be used as a driving parameter. For more advanced tests, evaluation of the shape and size of the pattern image was also tested. A piezoelectric actuator MIPOS250 from Piezo Jena was used with a range of $250 \mu \mathrm{m}$. A simple circular aperture was chosen as the test pattern with a diameter of $100 \mu \mathrm{m}$. The projection system de-magnifies the aperture $25 \times$ and thus the real size of the projected aperture is $4 \mu \mathrm{m}$. In Figure 7, there are images of the aperture on the photo-resist surface for two different positions of the microscope objective together with exposed images projected from the modulator.

One of the important advantages of laser writing techniques is the possibility of exposing the pattern at different depths of a recording material, when the depth of focus of the projected image is sufficiently low. For this purpose, the piezoelectric actuator which drives the microscope objective can be used for changing the position of the focal plane, while the focusing system is still applicable for stabilization the focus and deter- 


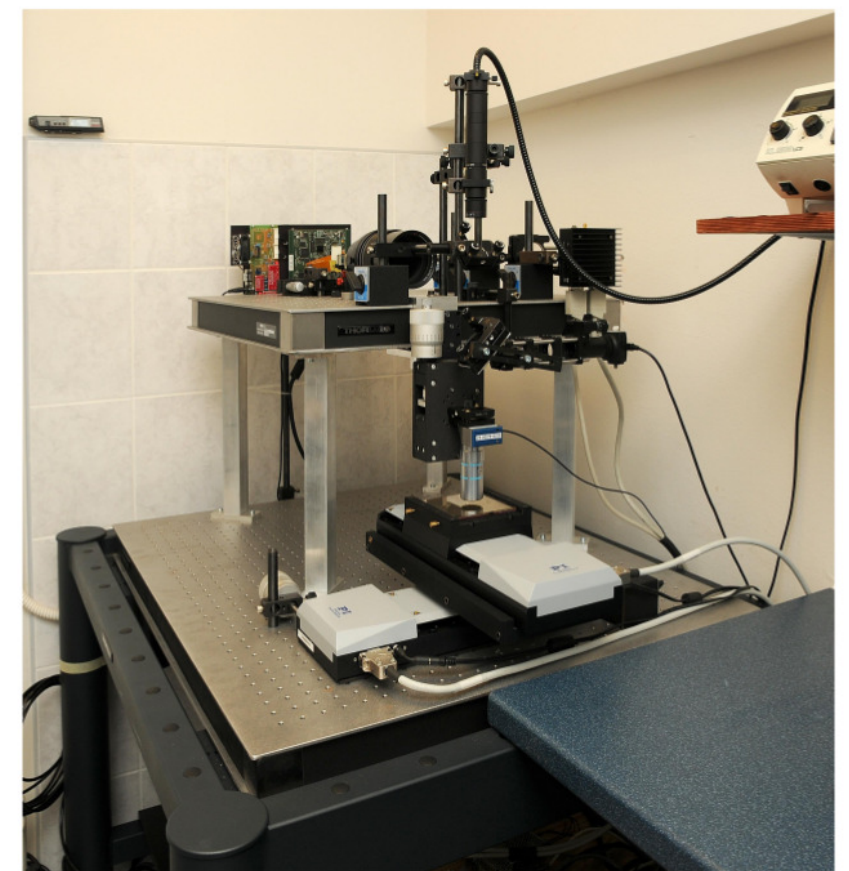

FIG. 8 Photography of the device. The whole assembly is placed on a dynamic vibration isolation table. The device works in a normal room environment.

mining the current position of the focal plane in the sample.

In F igure 8 there is an image of the system. The device consists of two platforms. The upper platform contains most of the optical elements, the lower platform includes only the stage and positioning system. Between the platforms are the microscope objective and the focusing system. The whole setup is placed on an active vibration isolation system ScienceDesk from Thorlabs. The exposure is driven directly from a PC via the custom-designed software interface. The exposure speed is mainly limited by the sensitivity of the recording material and the necessary relaxation times. During the experiments, the exposure speed about $4 \mathrm{~cm}^{2} /$ hour was reached. This value is more or less independent of the micro-structure and can be further improved by increasing the laser power and by optimizing the driving software.

\section{APPLICATIONS AND EXAMPLES}

The developed device can write a wide range of microstructures for photonic applications. Because of the very precise positioning of the details within the modulator area, nanometer precision in the mechanical movement of the stages, and the general shape of the exposing field, this technique can produce very interesting patterns. Several samples of micro-structures were prepared for particular applications. They demonstrate the flexibility and wide applicability of the proposed writing technique. Most structures were exposed in a positive photo-resist recording material (Rohm\&Haas, S1800 G series) with a thickness varying from several hundreds of nanometres up to 3 micrometres. Some experiments were done also with negative recording materials such as SU8 and Ormocers (all exposed with the blue laser at wavelength $405 \mathrm{~nm}$ ). These materials are more suitable for volume exposure of three dimensional elements.

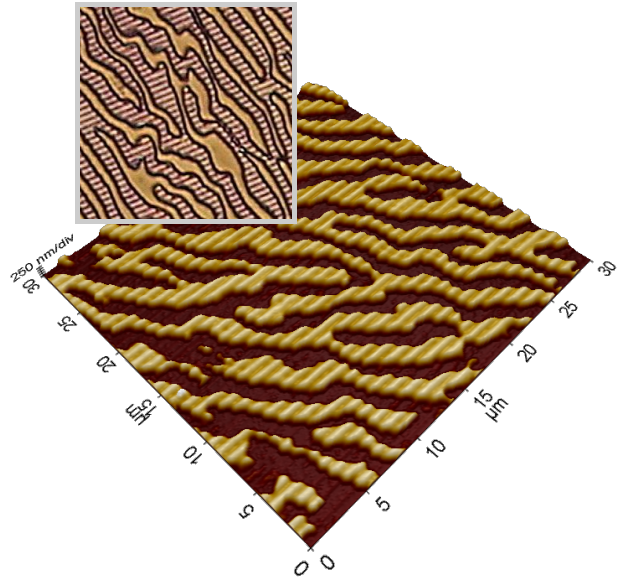

FIG. 9 An example of the aperiodic structure created using the matrix writing system. The AFM scan and the image from an optical microscope of the special synthetic hologram combined with a regular grating.

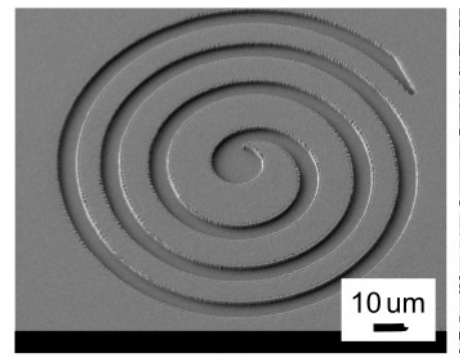

(a)

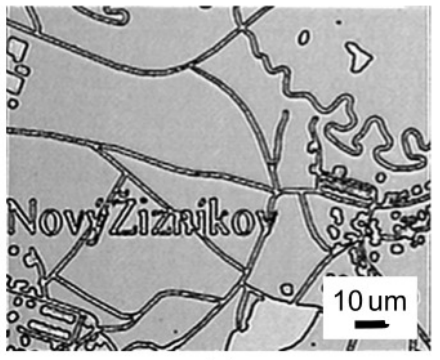

(b)
FIC. 10 Examples of general aperiodic structures created using the matrix writing system. In figure (a) there is a spiral micro-channel used for experiments with a selfassembly of micro-particles, which was originally realized in the photo-resist material and transfered to a thin plastic foil using an electro-forming and mechanical embossing processes. Figure (b) displays the magnified image of a micro-map exposed in a resist material.

In Figures 9 and 10 there are examples of aperiodic microstructures realized using the developed technique. As can be seen, complicated general shapes can be created. In Figures 11 and 12 there are examples of several periodic microstructures. Some of them were exposed using a single exposure, some were created using several subsequent exposures.

In Figure 13 there are examples of special structures prepared for application in optical micro-manipulations. The elements consist of smooth micro-channels with dimensions varying from 1 to 5 micrometres surrounded with micro-tips with high aspect ratio. The elements were used as substrates for micromanipulation with particles using optical tweezers (with artificially enhanced repulsiveness outside of the channels induced by the micro-tips).

\section{CONCLUSIONS}

The matrix laser lithography approach which uses the projection of the desired element or its parts from the computer driven spatial light modulator on the recording material was presented. The main principle of the technique, which was first used in synthetic image holography, was further devel- 
(a)

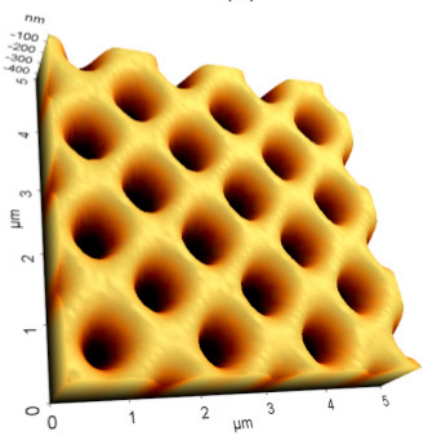

(c)

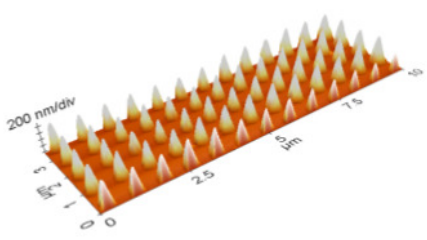

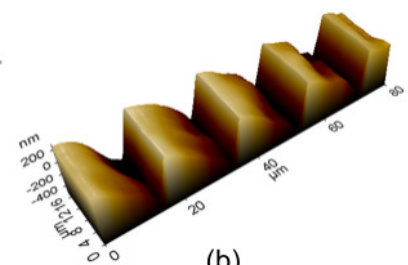

(b)

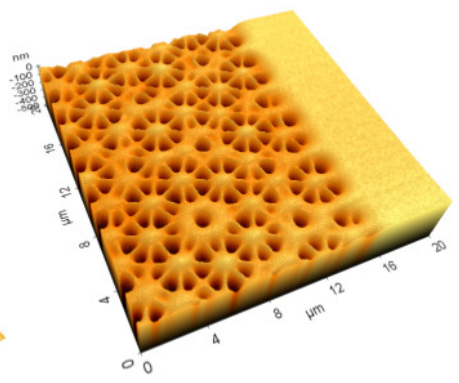

(d)
FIC. 11 Examples of various periodic structures exposed using the matrix writer in the resist material: AFM scans of (a) micro-tips, (b) a blazed-grating, (c) holes, and (d) a more complicated structure created using multiple exposures.

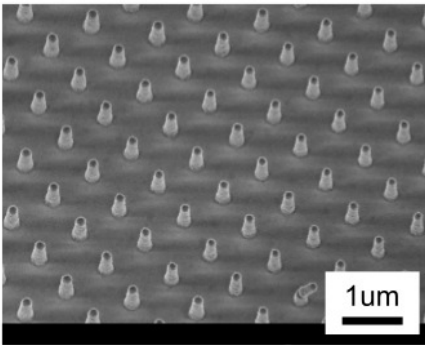

(a)

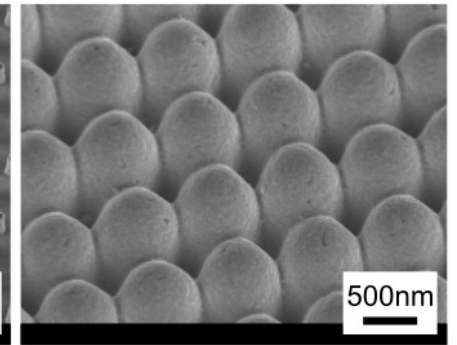

(b)
FIG. 12 SEM images of periodic micro-structures in (a) a photo-resist and (b) in a nickel layer.

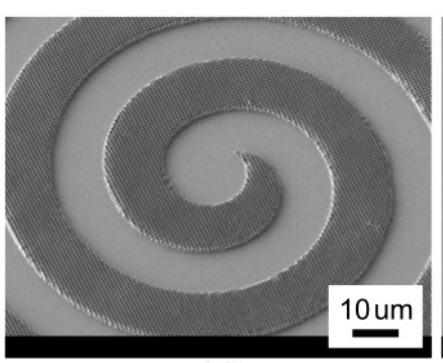

(a)

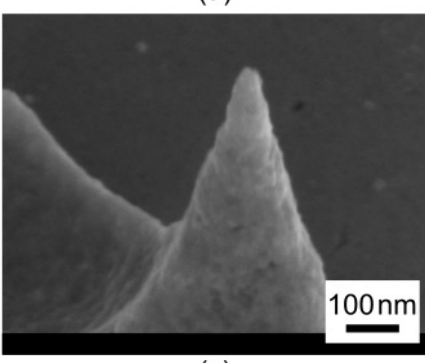

(c)

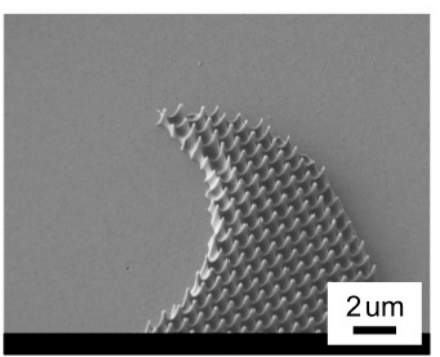

(b)

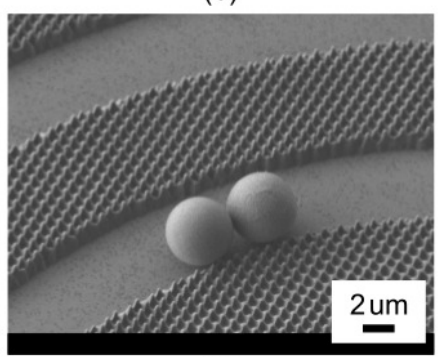

(d)
FIG. 13 Examples of special structures prepared for application in optical micromanipulations (a-c). The elements consist of smooth micro-channels with dimensions varying from 1 to 5 micrometres surrounded with high aspect micro-tips aligned in a periodic array. The elements were used as substrates for a micro-manipulation with particles using optical tweezers. An artificially enhanced repulsiveness was induced outside of the channels by the micro-tips. In figure (d) there is a substrate with $\mathrm{SiO}_{2}$ micro-spheres in the channel created. oped in order to improve the overall precision of the device and to enhance the flexibility of the writing process. The dynamic focusing system which is necessary when large areas are exposed and which can also serve as the tool for writing the elements with a three dimensional geometry was presented. Several examples which demonstrate the ability of the system to expose interesting elements for applications in optics, photonics, and other related fields were also presented.

\section{ACKNOWLEDGEMENTS}

Authors would like to acknowledge Jan Beránek for his help with the development and programming of electronic controllers. This work has been supported by the Grant Agency of Czech Academy of Sciences Project No. KAN401220801.

\section{References}

[1] R. F. Pease, and S. Y. Chou, "Lithography and other patterning techniques for future electronics," Proc. IEEE 96, 248-270 (2008).

[2] D. P. Sanders, "Advances in patterning materials for $193 \mathrm{~nm}$ immersion lithography," Chem. Rev. 110, 321-360 (2010).

[3] C. Wagner, and N. Harned, "EUV lithography: Lithography gets extreme," Nat.Photon. 4, 24-26 (2010).

[4] S. D. Gittard, and R. J. Narayan, "Laser direct writing of micro- and nano-scale medical devices," Expert Rev. Med. Devic. 7(3), 343-356 (2010).

[5] A. Ovsianikov, A. Ostendorf, and B. N. Chichkov, "Threedimensional photofabrication with femtosecond lasers for applications in photonics and biomedicine," Appl. Surf. Sci. 253, 6599-6602 (2007).

[6] A. Ovsianikov, M. Malinauskas, S. Schlie, B. Chichkov, S. Gittard, R. Narayan, M. Löbler, K. Sternberg, K. P. Schmitz, and A. Haverich, "Three-dimensional laser micro- and nano-structuring of acrylated poly(ethylene glycol) materials and evaluation of their cytoxicity for tissue engineering applications," Acta Biomater. 7(3), 947-974 (2011).

[7] D. Radtke, and U. D. Zeitner, "Laser-lithography on non-planar surfaces," Opt. Express 15(3), 1167-1174 (2007).

[8] G. Subramania, Y. J. Lee, A. J. Fischer, and D. D. Koleske, “LogPile $\mathrm{TiO}_{2}$ Photonic Crystal for Light Control at Near-UV and Visible Wavelengths," Adv. Mater. 22, 487-491 (2010).

[9] J. K. Gansel, M. Thiel, M. S. Rill, M. Decker, K. Bade, V. Saile, G. von Freymann, S. Linden, and M. Wegener, "Gold helix photonic metamaterial as broadband circular polarizer," Science 325 , 1513-1515 (2009).

[10] M. S. Rill, C. Plet M. Thiel, I. Staude, G. Von Freymann, S. Linden, and M. Wegener, "Photonic metamaterials by direct laser writing and silver chemical vapour deposition," Nat. Mater. 7, 543-546 (2008).

[11] C. W. J. Berendsen, M. Škereň, D. Najdek, and F. Černý, “Superhydrophobic surface structures in thermoplastic polymers by interference lithography and thermal imprinting," App. Surf. Sci. 255(23), 9305-9310 (2009).

[12] M. Altissimo, "E-beam lithography for micro/nanofabrication," Biomicrofluidics 4, 3-6 (2010).

[13] R. Menon, A. Patel, D. Gill, and H. I. Smith, "Maskless Lithography," Mater. Today 8, 26-33 (2005). 
[14] M. Campbell, D. N. Sharp, M. T. Harrison, R. G. Denning, and A. J. Turber, "Fabrication of photonic crystals for the visible spectrum by holographic lithography," Nature 404, 53-56 (2000).

[15] W. J. Daukscher, D. Mancini, K. Nordquist, D. J. Resnick, P. Hudek, D. Beyer, T. R. Groves, and 0. Fortagne, "Fabrication of Step-andflash Imprint Lithography Templates using a Variable Shaped Beam Exposure Tool," Microelectron. Eng. 75(4), 345-351 (2004).

[16] V. Mizeikis, V. Purlys, D. Paipulas, L. Maigyte, and K. Staliunas, "Tailoring of photonic structures by femtosecond laser lithography," Proc. SPIE 8204, 820417 (2011)

[17] M. Thiel, J. Fischer, G. von Freymann, and M. Wegener, "Direct laser writing of three-dimensional submicron structures using a continuous-wave laser at 532 nm," Appl. Phys. Lett. 97, 221102 (2010).

[18] T. Wang, W. Yu, D. Zhang, C. Li, H. Zhang, W. Xu, Z. Xu, H. Liu, Q. Sun, and Z. Lu, "Lithographic fabrication of diffractive optical elements in hybrid sol-gel glass on 3-D curved surfaces," 0 pt. Express 18, 25102-25107 (2010).

[19] S. K. Selvaraja, P. Jaenen, W.Bogaerts, D. Van Thourhout, P. Dumon, and R. Baets, "Fabrication of Photonic Wire and Crystal Circuits in Silicon-on-Insulator Using 193nm Optical Lithography," J. Lightwave Technol. 0, 1-8 (2009).

[20] A. G. Poleshchuk, A. A. Kutanov, V. P. Bessmeltsev, V. P. Korolkov, R. V. Shimanskii, A. I. Malyshev, A. E. Matochkin, N. V. Goloshevskii, K. V. Makarov, and V. P. Makarov, "Microstructuring of optical surfaces: Technology and device for direct laser writing of diffractive structures," Optoel. Instr. Data Proces. 6(2), 171-180 (2010).

[21] A. Bulanovs, E. Tamanis, and I. Mihailova, "Holographic recording device based on LCOS spatial light modulator," Latvian Journal of Phys. and Tech. Sciences 48(5), 60-68 (2011).
[22] M. Tang, Z. C. Chen, Z. Q. Huang, Y. S. Choo, and M. H. Hong, "Maskless multiple-beam laser lithography for large area nanostructure/microstructure fabrication," Appl. Opt. 50(35), 6536-6542 (2011).

[23] K. Obata, J. Koch, U. Hinze, and B. N. Chichkov, "Multi-focus twophoton polymerization technique based on individually controlled phase modulation," Opt. Express 18(16), 17193-17200 (2010).

[24] S. D. Gittard, A. Nguyen, K. Obata, A. Koroleva, R. J. Narayan, and B. N. Chichkov, "Fabrication of microscale medical devices by twophoton polymerization with multiple foci via a spatial light modulator," Biomed. Opt. Express 2(11), 3167-3178 (2011).

[25] T. Sandstrom, P. Askebjer, J. Sallander, R. Zerne, and A. Karawajczyk, "Pattern generation with SLM imaging," Proc. SPIE 4562, 38-44, (2002).

[26] L. H. Erdmann, A. Deparnay, F. Wirth, and R. Brunner, "MEMSbased lithography for the fabrication of micro-optical components," Proc. SPIE 5347, 79 (2004).

[27] L. Erdmann, A. Deparnay, G. Maschke, M. Langle, and R. Brunner, "MOEMS-based lithography for the fabrication of micro-optical components," J. Microlith. Microfab. 4, 041601 (2005).

[28] X. Zhu, Y. Xu, and S.Yang, "Distortion of 3D SU8 Photonic Structures Fabricated by Four-beam Holographic Lithography with Umbrella Configuration," Opt. Express 15(25), 16546-16560, (2007).

[29] I. Divliansky, and T. S. Mayer, "Fabrication of three-dimensional polymer photonic crystal structures using single diffraction element interference lithography," Appl. Phys. Lett. 82(11), 1667-1669 (2003).

[30] D. Pizzanelli, "The development of direct-write digital holography," Technical review at holographer.org (2004). 